\title{
Assessment of Biohostability of Clear Plastic and Metal Wire Retainers Used In Orthodontic Patients- An in Vitro Study
}

\author{
1sowbaraniya S. M', Muralidharan N. $\mathrm{P}^{2}$ and Aravind Kumar Subramanian ${ }^{3}$ \\ ${ }^{1}$ Saveetha Dental College and Hospitals Saveetha Institute of Medical and Technical \\ Sciences (SIMATS)Saveetha University Chennai-600077, Tamil Nadu, India. \\ ${ }^{2}$ Associate professor Department of Microbiology Saveetha Dental College and Hospitals Saveetha Institute of \\ Medical and Technical Sciences (SIMATS) Saveetha University, Chennai-600077, Tamil Nadu, India. \\ ${ }^{3}$ Professor Department of orthodontics Saveetha Dental College and Hospitals Saveetha Institute of Medical \\ and Technical Sciences (SIMATS) Saveetha University, Chennai-600077, Tamil Nadu, India.
}

\section{ABSTRACT}

To assess the biohostability of clear plastic and metal wire retainers used in orthodontic treatment which is done in vitro method. In this study, 2 groups of retainers were tested. Group 1 is Clear plastic retainers and group 2 is metal retainers. In each group 5 retainers, pieces were taken and the test is done along with controls. Metal wire retainers and clear plastic retainers are sterilized in an autoclave. In a conical flask $50 \mathrm{ml}$ of artificial saliva is taken and $1 \mathrm{~g}$ of glucose was added and it is sterilized. To this $100 \mu \mathrm{l}$ of S.mutans suspension was added. The sterilized artificial saliva with S. mutants was added to 2 test tubes. The retainers are taken in separate test tubes in which it contains 5 clear plastic retainers with $1 \mathrm{ml}$ of artificial saliva in each and another test tube contains 5 metal wire retainers with $1 \mathrm{ml}$ of artificial saliva and it is incubated for 24 hours. After 24 hours the retainers were rinsed with sterile normal saline and each retainer is placed separately in a sterilized cuvette containing $0.5 \mathrm{ml}$ of sterile saline. The cuvette is agitated turncoat the bacteria attached to it. Then a standard quantity was pipetted out onto a solid culture media and incubated at $37^{\circ} \mathrm{C}$. After the incubation, the plates were checked for their growth. Then the total colony-forming units were enumerated and the values were tabulated. It was observed that biohostability of clear plastic is comparatively less and the mean value graph obtained based on the bacterial growth is comparatively less in clear plastic whereas in metal wire retainers it's five times more than that of clear plastic. This was in the ratio of 1:5 ratio. Clear plastic has only the mean value of range 123.8 whereas the metal wire retainer has the mean value of range 669 which is five times more than that of clear plastic retainer. In this study, we can conclude that clear plastic retainer is comparatively less prone to bacterial colonization, whereas the metal wire retainer was more prone to bacteria colonization and the biohostability is high. As found in this study, the usage of clear plastic will help in maintaining better oral hygiene and for better aesthetics.

KEY WORDS: MALOCCLUSION , PLASTIC RETAINER, METAL WIRE RETAINER, BACTERIA.

\section{ARTICLE INFORMATION}

*Corresponding Author: muralidharan@saveetha.com

Received 7th Aug 2020 Accepted after revision 23rd Sep 2020

Print ISSN: 0974-6455 Online ISSN: 2321-4007 CODEN: BBRCBA

Thomson Reuters ISI Web of Science Clarivate Analytics USA and Crossref Indexed Journal

\section{Clarivate
Analytics}

NAAS Journal Score 2020 (4.31) SJIF: 2020 (7.728)

A Society of Science and Nature Publication,

Bhopal India 2020. All rights reserved.

Online Contents Available at: http//www.bbrc.in/

Doi: http://dx.doi.org/10.21786/bbrc/13.8/153 


\section{INTRODUCTION}

Malocclusion is a condition in which there is a deflection from the normal relation to the teeth to other teeth in the same arch or to the teeth in the opposing arch. Although malocclusion is itself neither a disease nor a life-threatening condition, there has been long marked demand for orthodontic treatment of malocclusion, placing a considerable role in dentistry(Bayat et al., 2016). The main indication for orthodontic treatment is of aesthetic value and general dental health. The main aim of orthodontic treatment is to eliminate functional problems(Harrison, 2011). Morphological stability is one of the important goals after orthodontic treatment from the patient's point of view. Relapse tendency for the teeth to return towards their pretreatment positions is more common without application of retainers(Yousif, 2014). Retention procedures are considered necessary to maintain the corrected positions of teeth(Valiathan and Hughes, 2010).

The main factors related to orthodontic retention failures are hereditary influences, disrespect to biological limits of dental movement and the use of removable and fixed retainers(Gunay and $0 z, 2018$ ). There are different types of retainers, fixed retainers and removable retainers. Fixed retainers are thin metal wire of less diameter run along the lingual or palatal surface of the teeth, which is bonded to teeth in certain places by cement and the removable retainer is a combo of metal wire and acrylic material that is inserted in and around the tooth it is instructed only if no movement is detected(Baka and Akın, 2017).

Permanent damage to tooth enamel can occur if the teeth and retainer are not kept clean. Areas on the enamel surface may begin to lose minerals leaving the unsightly white spots, leading to other problems like gingivitis development and leading to other severe problems. A large ratio of the disease can be prevented by practising proper oral hygiene techniques by providing oral health-related education thus improving oral attitude. For example, proper brushing is essential for cleaning teeth and gums effectively(Nene, 2014). Insufficient cleaning will lead to microbe's growth in retainers. The mostly found microbes are candida and staphylococcus that may include MRSA strains. Once the biofilms are formed in the retainer it's quite difficult to remove that biofilm which shows resistance to antimicrobials and difficult to clear(Chugh et al., 2019). The survival rate of retainers was reported over 12 to 24 months. In terms of retainer material, one study found metal wire fixed retainer and esthetic retainers made up of plastic have no difference in survival rate (Patel, Naini and Gill, 2013). The previous study has found that the thickness of wire is directly related to the thermoplastic fixed retainers and the number of teeth bonded(Littlewood et al., 2016).

Staphylococcus aureus is most commonly found on the retainers and tends to cause bloodstream infections which can lead to serious other infections(Ashwin and Muralidharan, 2015). So, it's important to maintain good oral hygiene. Acinetobacter baumanni, a critical pathogen least commonly found in oral cavities, tend to cause opportunistic infections. Although reports on A. baumannii as a dental pathogen is minimal, the propensity of the pathogen to evolve with a drug-resistant armour underscores the need for more research on this pathogen and its role in oral infections(Girija et al., 2019). A study suggested that herbal mouthwash plays an important role in maintaining good oral prophylaxis which leads to the extended survival rate of retainers(Selvakumar and Np, 2017). Patients undergoing orthodontic procedure have a common bacterial presence of spirochetes because they can easily undergo rapid multiplication and cause severe bacterial infection((Website, no date).

Enterococcus faecalis, the predominant human enterococcus is the main cause for varies oral infections such as caries, peri-implantitis, endodontic failure, periodontitis and leads to severe other infections and by good maintenance of oral hygiene the colonisation of bacteria can be reduced (Marickar, Geetha and Neelakantan, 2014). Orange peel oil has a good antibacterial activity against the streptococcus mutans which is the major reason for causing caries and other infections(Vaishali and Geetha, 2018). Laurus nobilis L. essential oils are able to inhibit oral S. aureus strains with important antibiofilm efficacy(M, Geetha and Thangavelu, 2019). It could have a promising role in the prevention of oral diseases.A.baumannii may be present in oral biofilms, which may act as a reservoir for pneumonia and chronic obstructive pulmonary disease. Subgingival colonization by A. baumannii increases the risk of refractory periodontitis(Vijayashree Priyadharsini, Smiline Girija and Paramasivam, 2018).

Acinetobacter was considered an opportunistic agent of low pathogenicity and number of virulence factors have been described that make it possible to survive in the hospital setting and the ability to cause disease, particularly in debilitated patients(Girija, Jayaseelan and Arumugam, 2018).m6Ais useful in preventing various oral infections and maintains the normal flora of oral cavity(Paramasivam, Vijayashree Priyadharsini and Raghunandhakumar, 2020).A.baumannii a nosocomial pathogen enter into oral cavity in association with other oral pathogens it tends to cause aggressive periodontitis(Priyadharsini et al., 2018).A study suggested that Silicon analysis of genotype G1-G4 can be used as candidates for developing novel therapeutic drugs against oral pathogens(Smiline, Vijayashree and Paramasivam, 2018). An article suggested that Acacia nilotica has a good antibacterial effect and can be used for maintaining good oral hygiene(Shahzan et al., 2019).

\section{MATERIAL AND METHODS}

In this study 2 groups of retainers were tested. Group 1 is Clear plastic retainers and the group 2 is metal retainers. The retainers were cut into small pieces of uniform length of $1 \mathrm{~cm}$ In each group 5 retainers pieces were taken and the test was done along with controls. Clear plastic and metal wire retainers are packed in such a way that it 
withstands high temperature to undergo the autoclave process. Metal wire retainers and clear plastic retainers are sterilized in autoclave. In the autoclave process the metal wire and clear plastic retainers are subjected to 15 lbs pressure for 30 minutes.

In a conical flask $50 \mathrm{ml}$ of artificial saliva is taken and $1 \mathrm{~g}$ of glucose was added and it is sterilized by autoclaving. To this $100 \mu \mathrm{l}$ of S.mutans suspension was added. I ml of the sterilized artificial saliva with $\mathrm{S}$. mutans was added to 2 test tubes. The retainers are taken in separate test tubes in which it contains 5 clear plastic retainers with $1 \mathrm{ml}$ of artificial saliva. After placing the retainers the tubes were incubated at $37^{\circ} \mathrm{C}$ for 24 hours. After 24 hours the retainers were rinsed with sterile normal saline and each retainer is placed separately in a sterilized cuvette containing $0.5 \mathrm{ml}$ of sterile saline. Cuvette is agitated to uncoat the bacteria attached to it. Then a standard quantity of $10 \mu \mathrm{l}$ was pipetted out onto a solid culture media and incubated at $37^{\circ} \mathrm{C}$. The solid culture medium chosen for the study is Nutrient agar (Himedia). Nutrient agar is a general purpose nutrient medium used for the cultivation of microbes supporting growth of many nonfastidious organisms. Nutrient agar can grow the variety of bacteria and contains many nutrients needed for the bacterial growth. After the incubation the plates were checked for their growth. Then the total colony forming units were enumerated and the values were tabulated.

Figure 1: Plastic wire retainer

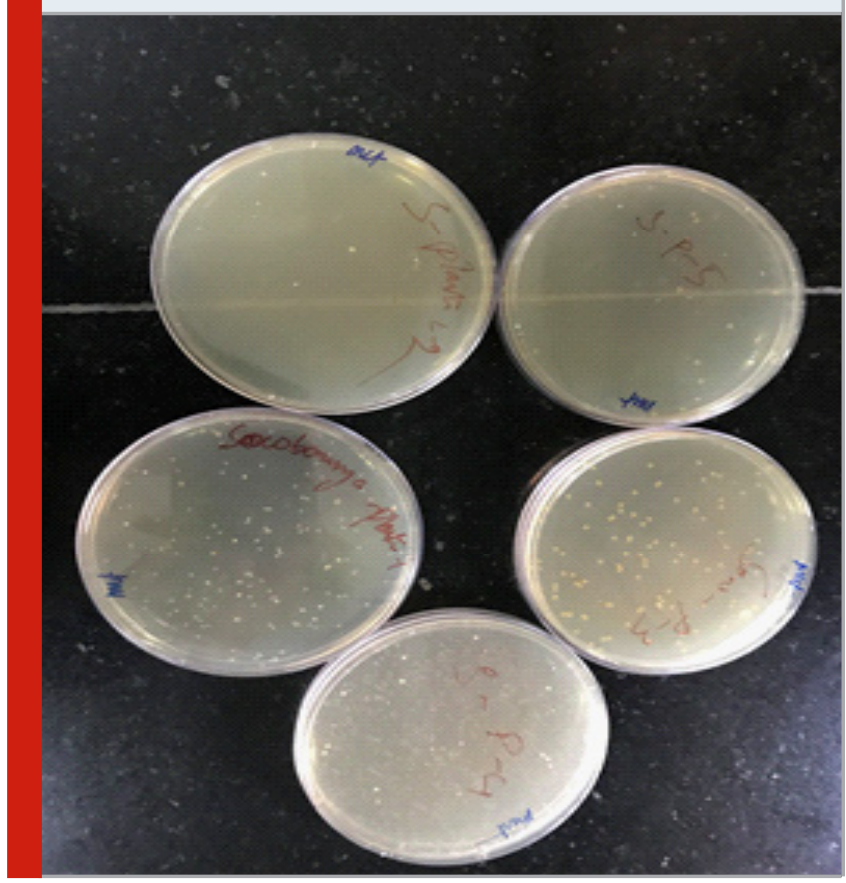

RESULTS AND DISCUSSION

After 24 hours of incubation the bacteria formed in the Nutrient agar was counted and the mean value was obtained which was counted manually. After 24 hours the mean value of bacteria present in the clear plastic is $144 \mathrm{CFU}$, whereas in the metal the mean CFU value was 669 which was comparatively high, though same temperature and same ph is provided for both clear plastic and metal retainers, metal wire is more prone to bacteria attachment and the more bacteria is formed in metal retainers. Biohostibility ratio was 1: 5 in Clear plastic and metal wire retainers. The control group were kept there will be no bacterial growth because Streptococcus mutans were not inoculated. The control group was used for the reference to differentiate after each process and the changes in the samples taken for the easy comparison.

Figure 2: Metal wire retainer

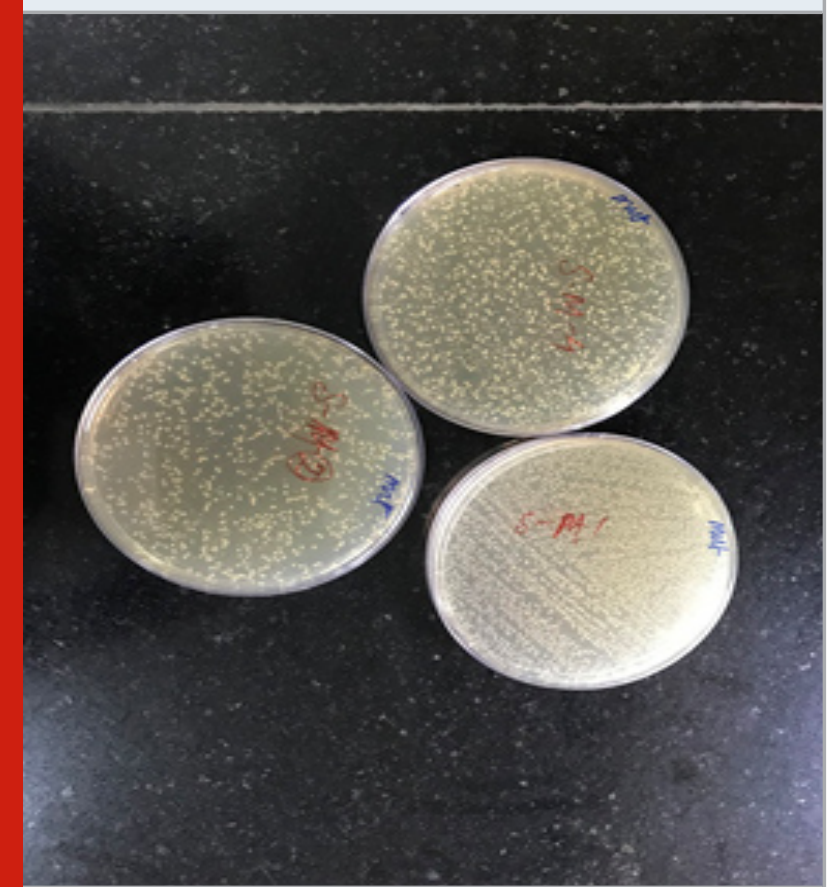

Table 1 . The table depicts the mean value of the colony forming bacteria.

\begin{tabular}{|l|c|c|}
\hline & $\begin{array}{c}\text { Plastic } \\
\text { retainer }\end{array}$ & $\begin{array}{c}\text { Metal wire } \\
\text { retainer }\end{array}$ \\
\hline $\begin{array}{l}\text { No.Of.Colony } \\
\text { forming Bacteria } \\
\text { present }\end{array}$ & 619 & 3348 \\
\hline Mean Value & 123.8 & 669 \\
\hline
\end{tabular}

Plate shows the colony forming bacteria in the plastic and metal wire retainer after $24 \mathrm{hrs}$ of incubation (fig $1 \mathrm{ct} 2$ ).

From the above mentioned table, the mean value of the plastic retainer was 123.8 whereas metal retainer was 669 which was nearly five times of the plastic retainer value.

From the above mentioned graph we can see that there is wide variation in the mean value of the bacterial attachment and colonization. Clear plastic has a mean value of 123.8 whereas the metal wire retainer has the mean value of 669 which is five times more than that 
of clear plastic retainer. The wide variation in the graph is due to that metal wire retainers attach to the bacteria easily and if not properly cleaned, counter tops the appliance can lead to colony forming of bacteria and leads to many bacterial diseases. But incase of clear plastic retainer bacteria is not as much as attached to the plastic surface so the adherence of the bacteria is less and the number of bacteria growing in the clear plastic retainer is comparatively less than the metal retainer.

Figure 3: The graph depicts the mean value graph of the colony forming unit of the bacteria in clear plastic and metal wire retainers

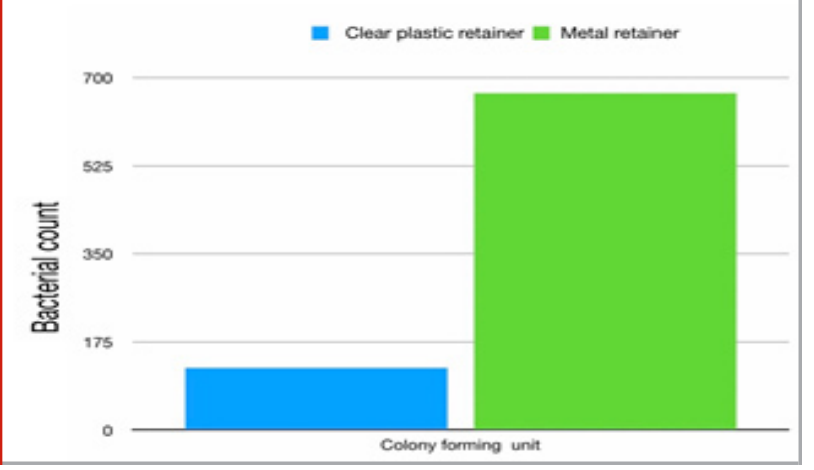

From the previous study, it was said that clear plastic retainer's survival rate was reported less than the metal wire(Wiegand and Kanzow, 2020).Moreover metal wire retainers have more growth of S.mutans because it is normally present in oral cavities and this facultative anaerobes grow more in the metal retainers than in the clear plastic.Clearly, the biohostability of metal wire retainers is comparatively more due to the environment and the $\mathrm{pH}$ variation in the oral cavity(Scribante et al., 2011). So, to reduce the bacteria growth in the metal retainer's usage of antibacterial toothpaste which will reduce the viability and the adhering of. Bacteria in the tooth surface (Thickett and Power, 2010).Mostly commonly toothpaste containing sodium lauryl sulphate and stannous fluoride or triclosan will increase the ph of oral biofilms and decrease the viability of bacteria(Wessel et al., 2014).oil containing mouth rinses remove the bacteria from the oral cavity through bacterial adhesion to the hydrophobic oil(Bedran et al., 2014).

Though the clear plastic retainer has more advantages associated, one of the main criteria is it has a very good aesthetic compared to metal wire retainer. Though the Biohostibility plays a vital role in harboring the bacteria on their surface, maintaining a good oral hygiene is pivotal in extracting maximum benefit in using a prosthetic material. Most of the better results are associated with periodontal health of the patients and how the patients maintain the oral health(Nalçacı et al., 2014). The main failure associated with metal wire retainer is not only the bacterial growth other reasons include such as poor aesthetics and the duration of follow up. Though clear plastic also needs to be followed up, its comparatively less follow up required than the metal wire retainer(Levrini et al., 2015).
Though clear plastic has many advantages associated the major drawback is survival rate it is approximately 12 -24 months which is comparatively less than the metal wire retainer(Salehi, Najafı and Roeinpeikar, 2013).The use of clear plastic is much more convenient than the metal wire retainer because metal wire requires more oral hygiene or else poor hygiene will lead to mass growth of bacteria. Comparatively bonded retainers will have more better results than the removable retainers in both clear plastic and metal wire retainers(Dietrich et al., 2015). But the major disadvantage associated with the bonded retainer is depends on the periodontal health patients so for the people with weak periodontal ligament it directly affect the outcome of treatment, so it's very important to consider the periodontal health of the Patients.

\section{CONCLUSION}

According to this study it can be concluded that clear plastic retainer is comparatively less prone to bacteria attachment, whereas the metal wire retainer was more prone to bacteria attachment and the Biohostibility is high. So, maintaining a good oral hygiene and usage of oil mouth rinses and toothpaste containing sodium lauryl sulphate will decrease the viability and the adherence of the bacteria. So the usage of clear plastic will help in getting better results and for the better aesthetics and the main criteria associated is the usage of clear plastic retainer will help preventing the deterioration of oral health.

\section{Conflict of Interest: Nil}

\section{REFERENCES}

Ashwin, K. S. and Muralidharan, N. P. (2015)

'Vancomycin-resistant enterococcus (VRE) vs Methicillin-resistant Staphylococcus Aureus (MRSA)', Indian journal of medical microbiology, 33 Suppl, pp. 166-167. doi: 10.4103/0255-0857.150976.

Baka, Z. M. and Akın, M. (2017) 'A prospective clinical evaluation of fixed retainer failures', Acta Odontologica Turcica. doi: 10.17214/gaziaot.299307.

Bayat, J. T. et al. (2016) 'Predicting orthodontic treatment need: reliability and validity of the Demand for Orthodontic Treatment Questionnaire', The European Journal of Orthodontics, p. cjw056. doi: 10.1093/ejo/ cjw056.

Bedran, T. B. L. et al. (2014) 'Subinhibitory concentrations of triclosan promote Streptococcus mutans biofilm formation and adherence to oral epithelial cells', PloS one, 9(2), p. e89059. doi: 10.1371/journal. pone.0089059.

Chugh, V. K. et al. (2019) 'Survival rate of two orthodontic bonded retainer wires', American Journal of Orthodontics and Dentofacial Orthopedics, pp. 4-5. doi: 10.1016/j.ajodo.2018.09.009.

Dietrich, P. et al. (2015) 'Long-term follow-up of maxillary fixed retention: survival rate and periodontal 
health', European journal of orthodontics, 37(1), pp. 37-42. doi: 10.1093/ejo/cju001.

Girija, A. S. S. et al. (2019) 'Plasmid-encoded resistance to trimethoprim/sulfamethoxazole mediated by dfrA1, dfrA5, sul1 and sul2 among Acinetobacter baumannii isolated from urine samples of patients with severe urinary tract infection', Journal of Global Antimicrobial Resistance, pp. 145-146. doi: 10.1016/j. jgar.2019.04.001.

Girija, S. A. S., Jayaseelan, V. P. and Arumugam, P. (2018) 'Prevalence of VIM- and GIM-producing Acinetobacter baumannii from patients with severe urinary tract infection', Acta Microbiologica et Immunologica Hungarica, pp. 539-550. doi: 10.1556/030.65.2018.038.

Gunay, F. and Oz, A. A. (2018) 'Clinical effectiveness of 2 orthodontic retainer wires on mandibular arch retention', American journal of orthodontics and dentofacial orthopedics: official publication of the American Association of Orthodontists, its constituent societies, and the American Board of Orthodontics, 153(2), pp. 232-238. doi: 10.1016/j.ajodo.2017.06.019. Harrison, J. (2011) 'Orthodontic treatment', Vital, pp. 31-35. doi: 10.1038/vital1329.

Levrini, L. et al. (2015) 'Periodontal health status in patients treated with the Invisalign ${ }^{\circledR}$ system and fixed orthodontic appliances: A 3 months clinical and microbiological evaluation', European Journal of Dentistry, pp. 404-410. doi: 10.4103/13057456.163218.

Littlewood, S. J. et al. (2016) 'Retention procedures for stabilising tooth position after treatment with orthodontic braces', Cochrane Database of Systematic Reviews. doi: 10.1002/14651858.cd002283.pub4.

Marickar, R. F., Geetha, R. V. and Neelakantan, P. (2014) 'Efficacy of contemporary and novel Intracanal medicaments against enterococcus faecalis', The Journal of clinical pediatric dentistry, 39(1), pp. 47-50. doi: 10.17796/jcpd.39.1.wmw9768314h56666.

M, M. A., Geetha, R. V. and Thangavelu, L. (2019) 'Evaluation oEvaluation of anti-inflammatory action of Laurus nobilis-an in vitro studyf anti-inflammatory action of Laurus nobilis-an in vitro study', International Journal of Research in Pharmaceutical Sciences, pp. 1209-1213. doi: 10.26452/ijrps.v10i2.408.

Nalçac1, R. et al. (2014) 'Effect of bracket type on halitosis, periodontal status, and microbial colonization', The Angle Orthodontist, pp. 479-485. doi: 10.2319/061913461.1.

Nene, H. J. S. (2014) 'Does Text Messaging Reminder Help in the Orthodontic Compliance of Patients to Maintain their Oral Hygiene', Journal of Oral Hygiene Et Health. doi: 10.4172/2332-0702.1000152.
Paramasivam, A., Vijayashree Priyadharsini, J. and Raghunandhakumar, S. (2020) 'N6-adenosine methylation (m6A): a promising new molecular target in hypertension and cardiovascular diseases', Hypertension research: official journal of the Japanese Society of Hypertension, 43(2), pp. 153-154. doi: 10.1038/s41440019-0338-z.

Patel, A., Naini, F. B. and Gill, D. (2013) 'Bonded orthodontic retainers', Orthodontic Update, pp. 70-77. doi: 10.12968/ortu.2013.6.3.70.

Priyadharsini, J. V. et al. (2018) 'An insight into the emergence of Acinetobacter baumannii as an oro-dental pathogen and its drug resistance gene profile - An in silico approach', Heliyon, p. e01051. doi: 10.1016/j. heliyon.2018.e01051.

Salehi, P., Najafi, H. Z. and Roeinpeikar, S. M. (2013) 'Comparison of survival time between two types of orthodontic fixed retainer: a prospective randomized clinical trial', Progress in Orthodontics, p. 25. doi: 10.1186/2196-1042-14-25.

Scribante, A. et al. (2011) 'Efficacy of Esthetic Retainers: Clinical Comparison between Multi Strand Wires and Direct-Bond Glass Fiber-Reinforced Composite Splints', International Journal of Dentistry, pp. 1-5. doi: 10.1155/2011/548356.

Selvakumar, R. and Np, M. (2017) 'COMPARISON IN BENEFITS OF HERBAL MOUTHWASHES WITH CHLORHEXIDINE MOUTHWASH: A REVIEW', Asian Journal of Pharmaceutical and Clinical Research, p. 3. doi: 10.22159/ajpcr.2017.v10i2.13304.

Shahzan, M. S. et al. (2019) 'A computational study targeting the mutated L321F of ERG11 gene in C. albicans, associated with fluconazole resistance with bioactive compounds from Acacia nilotica', Journal de Mycologie Médicale, pp. 303-309. doi: 10.1016/j. mycmed.2019.100899.

Smiline, A. S. G., Vijayashree, J. P. and Paramasivam, A. (2018) 'Molecular characterization of plasmid-encoded blaTEM, blaSHV and blaCTX-M among extended spectrum $\otimes$-lactamases [ESBLs] producing Acinetobacter baumannii', British Journal of Biomedical Science, pp. 200-202. doi: 10.1080/09674845.2018.1492207.

Thickett, E. and Power, S. (2010) 'A randomized clinical trial of thermoplastic retainer wear', The European Journal of Orthodontics, pp. 1-5. doi: 10.1093/ejo/ cjp061.

Vaishali, M. and Geetha, R. V. (2018) 'Antibacterial activity of Orange peel oil on Streptococcus mutans and Enterococcus-An In-vitro study', Research Journal of Pharmacy and Technology, p. 513. doi: 10.5958/0974360x.2018.00094.x.

Valiathan, M. and Hughes, E. (2010) 'Results of a survey-based study to identify common retention 
practices in the United States', American Journal of Orthodontics and Dentofacial Orthopedics, pp. 170-177. doi: 10.1016/j.ajodo.2008.03.023.

Vijayashree Priyadharsini, J., Smiline Girija, A. S. and Paramasivam, A. (2018) 'In silico analysis of virulence genes in an emerging dental pathogen A. baumannii and related species', Archives of oral biology, 94, pp. 93-98. doi: 10.1016/j.archoralbio.2018.07.001.

Website (no date). Available at: Shahana RY, Muralidharan NP. Efficacy of mouth rinse in maintaining oral health of patients attending orthodontic clinics [Internet]. Vol. 9, Research Journal of Pharmacy and Technology. 2016. p. 1991. Available from: http://dx.doi.org/10.5958/0974- 360x.2016.00406.6 (Accessed: 29 June 2020). Wessel, S. W. et al. (2014) 'Adhesion Forces and Composition of Planktonic and Adhering Oral Microbiomes', Journal of Dental Research, pp. 84-88. doi: 10.1177/0022034513511822.

Wiegand, A. and Kanzow, P. (2020) 'Effect of Repairing Endodontic Access Cavities on Survival of Single Crowns and Retainer Restorations', Journal of endodontia, 46(3), pp. 376-382. doi: 10.1016/j.joen.2019.11.012.

Yousif, A. (2014) 'Four years stability evaluation of the changes obtained by twin force bite corrector appliance', Egyptian Orthodontic Journal, pp. 49-74. doi: 10.21608/ eos.2014.78898. 